\title{
Extranodal Large B-Cell Type Aggressive Non-Hodgkin's Lymphoma
}

\author{
${ }^{1}$ Sonal Gupta, ${ }^{2}$ Parvind Gumber
}

\begin{abstract}
Non-Hodgkin's lymphomas belongs to a group of neoplasm, originate from the cells of lymphoreticular system that shows diversity in manner of presentation, response to therapy and prognosis. Non-Hodgkin's lymphoma accounts for $80-90 \%$ of all lymphomas. Though primary intraoral lesion in nonHodgkin's lymphoma are uncommon, it is important to be aware of them since intraoral manifestation are presenting symptom in these patients. A 72-year-old Indian male presented with complaint of asymptomatic swelling in the left side of face since one and half months. A careful clinical evaluation supported by radiologic, histopathologic and immunohistochemical investigations will help in identifying the disease at an early stage, which will result in better prognosis.
\end{abstract}

Keywords: Extranodal, Lymph nodes, Non-Hodgkin's lymphoma, Swelling.

How to cite this article: Gupta S, Gumber P. Extranodal Large B-Cell Type Aggressive Non-Hodgkin's Lymphoma. J Mahatma Gandhi Univ Med Sci Tech 2016;1(2):71-74.

\section{INTRODUCTION}

Lymphoma is a malignant neoplasm that account for $2.6 \%$ of all malignant neoplasm. ${ }^{1}$ It is second common malignancy of head and neck region next to squamous cell carcinoma. ${ }^{2}$ It comprises of more than 67 subtypes of two closely related cancer that affect the lymphatic system. Generally, lymphomas present as nodal disease, but may involve the extranodal sites, i.e., thymus, spleen, liver, gastrointestinal tract and oral cavity. Primary extranodal lymphomas account for up to $20-30 \%$ of all lymphomas. ${ }^{3}$

Non-Hodgkin's lymphoma is a heterogeneous group of malignancies most frequently occurring in older individuals with a mean age is 60 years. About one-third cases of non-Hodgkin's lymphoma commonly involve extranodal sites. ${ }^{4}$ Among them, 2-3\% of the cases are associated with the oral cavity. ${ }^{5}$ The most commonly

\footnotetext{
${ }^{1,2}$ Senior Lecturer

${ }^{1,2}$ Department of Oral and Maxillofacial Pathology and Microbiology, Mahatma Gandhi Dental College, Jaipur, Rajasthan, India

Corresponding Author: Parvind Gumber, Department of Oral and Maxillofacial Pathology and Microbiology, Mahatma Gandhi Dental College, Jaipur, Rajasthan, India, e-mail: dr.parvindgumber@yahoo.com
}

involve area in the oral cavity is the mucosa associated with Waldeyer's ring. ${ }^{6}$ According to National Cancer Institute, non-Hodgkin's lymphoma account for 55,000 to 66,000 new cases and approximately 19,000 deaths per year. $^{3}$

As oral non-Hodgkin's, lymphoma are rare in occurrence with a wide range of histologic appearance and clinical diagnosis. The present report describe a case of non-hodgkin lymphoma of extranodal site primarily involve buccal mucosa in 72-year-old male.

\section{CASE REPORT}

A 72-year-old man reported with the complaint of an asymptomatic swelling in the left side of face since one and half months. The swelling was insidious in onset, gradually increasing in size and attained the present size. The patient was hypertensive and was on medication for the past 10 years.

Extraoral examination revealed a $4 \times 3 \mathrm{~cm}$ smooth surface swelling involving left side of face extending to lower border of orbit superiorly and $1.5 \mathrm{~cm}$ above the corner of mouth inferiorly, medially swelling extend a few $\mathrm{cm}$ away from the ala of nose and laterally extend up to $3 \mathrm{~cm}$ in front of the ear. On palpation, swelling was hard and non tender.

Intraoral examination revealed $2 \times 1.5 \mathrm{~cm}$ swelling on left side of buccal mucosa opposite to left maxillary premolar area (Fig. 1). On palpation swelling was firm, non tender and was not associated with sinus or pus discharge. No palpable submandibular and cervical lymphadenopathy was evident.

\section{INVESTIGATION}

A paranasal sinus radiograph revealed the presence of mucosal thickening that was closely associated with left maxillary sinus. Computed tomography (CT) scan examination revealed the presence of a soft tissue lesion measuring $38 \times 30 \times 21 \mathrm{~mm}$ on left side of face (Fig. 2). The lesion appeared to be in muscular plane and the underlying bone was preserved. Nasal septum was mildly diverted toward right side, both the inferior turbinate was hypertrophied. Polypoidal mucosal thickening was seen in left maxillary sinus suggestive of benign soft tissue neoplasm. For further evaluation patient was subjected 


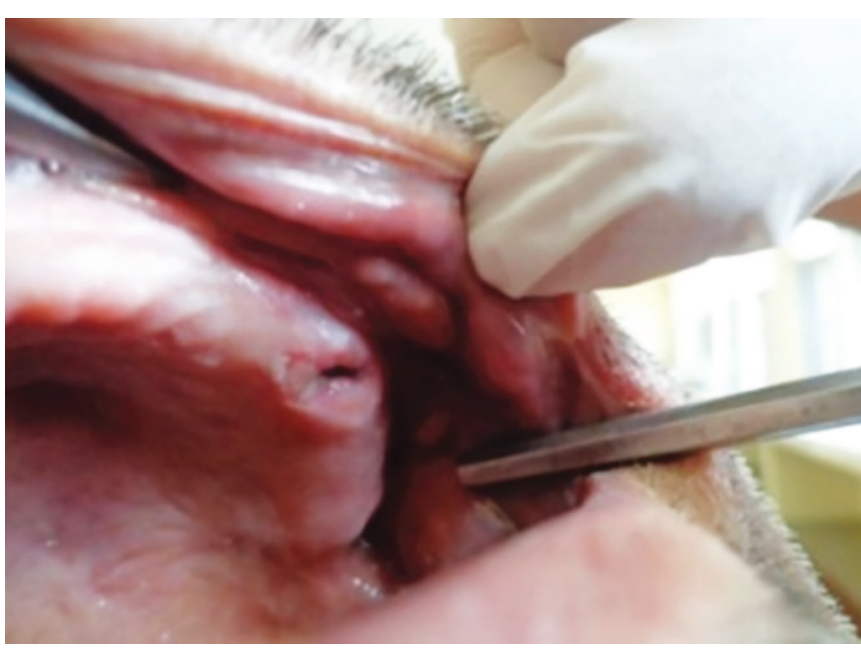

Fig. 1: Patient with swelling on left side of buccal mucosa opposite to premolar area

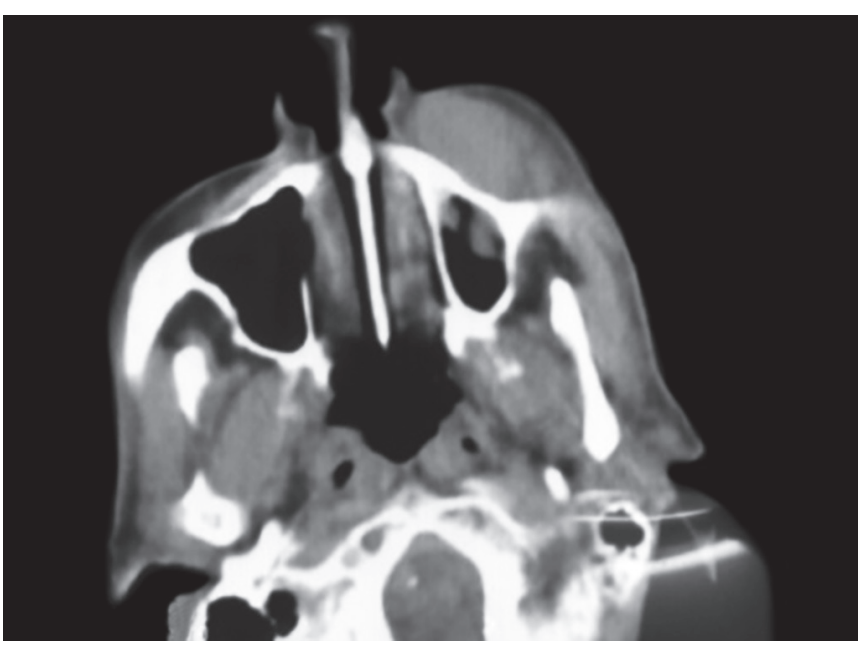

Fig. 2: Soft tissue lesion measuring $38 \times 30 \times 21 \mathrm{~mm}$ on anterior part in muscular plane of left cheek-inflammatory origin; mild nasal septum deviation to right side; bilateral inferior turbinates hypertrophy; polypoidal mucosal thickening in left maxillary sinus

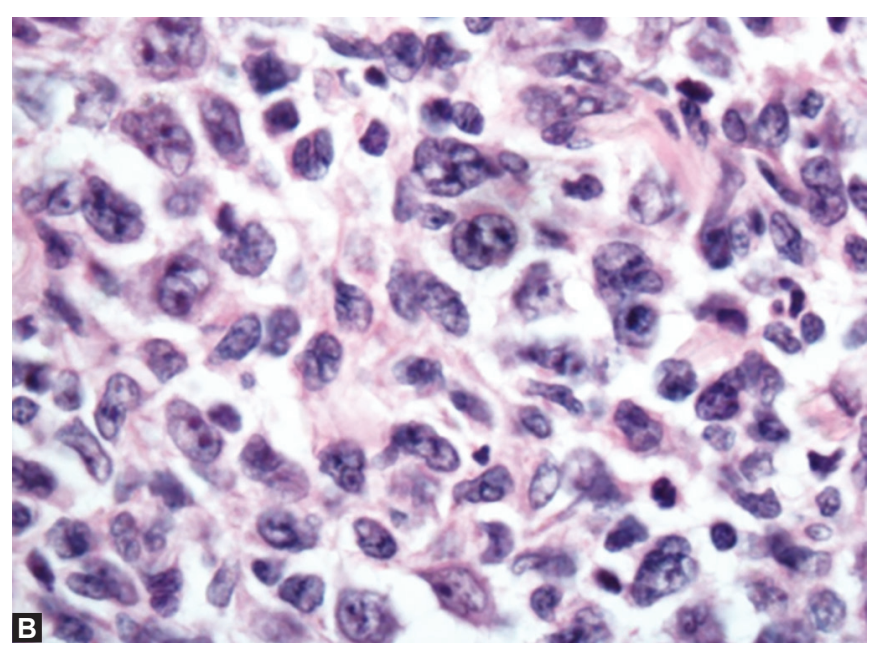

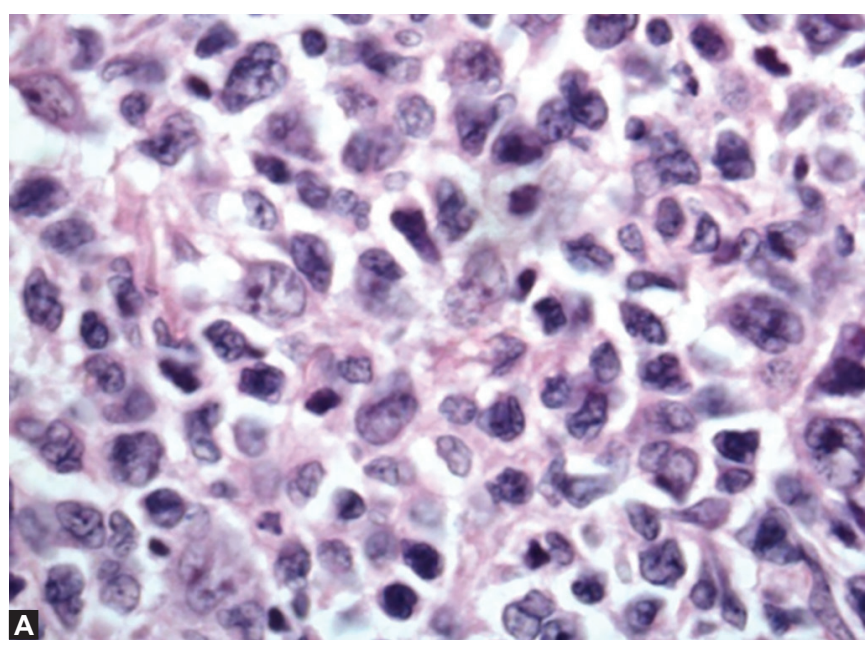

Figs 3A and B: (A) Presence of small and large malignant lymphocytic cells and (B) presence of large binucleated cells with pale cytoplasm and prominent nucleoli and few cells showing abnormal mitotic figures

for ultrasonography of left side of face which revealed the presence of small hypoechoic areas in the areas that was closely associated with the swelling region and also on color Doppler ultrasonography examination revealed that there was decrease in vascularity in that region. Differential diagnosis based on the above investigation suggestive of fungal granuloma, chronic granulomatous lesion, soft tissue malignancy, and lymphoma.

An incisional biopsy was carried out for histopathological examination. Grossly, the tissue was creamish white to light brown in color and was soft to firm in consistency. Light microscopic examination of section stained with H\&E revealed the presence of small and large malignant lymphocytic cell. The large lymphoid cells were 2-3 times larger than normal lymphocytic cells. Connective tissue stroma also shows the presence of large binucleated cell with pale cytoplasm and prominent nucleoli (Fig. 3A). Few areas also show the presence of immature large cell with cleave nucleus and presence of numerous abnormal mitotic figures was also noted (Fig. 3B). The histopathological features were suggestive of non-Hodgkin's lymphoma.

A panel of immunohistochemical marker was also used for confirmation and treatment plan. Immunohistochemistry revealed the lymphoid tumor cells were strongly positive for pan B cell marker (CD20) and mild positivity for T cell (CD-3) antigen. Typical large lymphoid cells were also strongly positive for (leukocyte common antigen CD-45), BCL-2 and negative for CD-30, which finally proved the lesion to be a diffuse large B cell non-Hodgkin's lymphoma. Further bone marrow biopsy showed normocellular marrow spaces with adequate representation of all hematopoietic marrow elements showing no signs of infilteration by 
non-Hodgkin's lymphoma. Positron emission tomography (PET) scan was done to assess the distribution of malignant cells. There was intensive uptake of fluorodeoxyglucose (FDG) in soft tissue lesion over the left maxilla extending mesially till the left nostril, posteriorly eroding the anterior wall of maxillary sinus and inferiorly up to lower eyelid. Intensive uptake of FDG was also seen in bilateral group of parotid lymph node and mild uptake was seen in left cervical lymph node and patient was grade to the level stage III according to Ann Arbor classification with the involvement of lymph node and contiguous extra lymphatic sites on both side of diaphragm.

\section{DISCUSSION}

Lymphoma is solid tumor of immune system that primarily involves the lymph node and rarely involves the extra lymphatic sites. Diagnosis of lymphomas is based on the WHO classification and broadly divided into two main categories. ${ }^{7}$

Non-Hodgkin's lymphoma covers a wide range of lymphoid neoplasia. The annual incidence rate of the individual diagnosed with non-Hodgkin's lymphoma has increased since the last few years. ${ }^{8}$ Extranodal nonHodgkin's lymphoma is a distinct entity and usually account for $40 \%$ of all non-Hodgkin's lymphoma. It was first described by Isaacson and Wright in $1983 .{ }^{9}$ The diagnosis of oral extranodal non-Hodgkin's lymphoma is challenging and may cause relevant problem with their differential diagnosis in view of rarity in the primary occurrence of non-Hodgkin's lymphoma in oral cavity and wide variation in their clinicoradiographic presentation and histologic features. Clinically, when non-Hodgkin's lymphoma first appear in the oral cavity they generally appear as non-tender, soft to firm swelling may or may not associated with ulceration. Usually in most of the cases patients present with clinical symptoms like night sweat, fever, weight loss, and palpable lymph nodes; but, in present case, no such clinical symptoms and palpable lymph nodes were evident which may often lead to misdiagnosed as squamous cell carcinoma, fungal infection or any other soft tissue tumor. ${ }^{10}$ So, further investigation must be done to rule out the possibility of theses lesions.

Conventional radiography examination did not reveal any significant difference, but with ultrasonography examination lymphnode enlargement in submandiblar and cervical area gave a significant finding which was not assessed clinically and proved that lesion may be associated with lymph nodes. Positron emission tomography examination further improve the accuracy of diagnosis by assessing the uptake of FDG in the region of lymph node and other extra lymphatic site and prove to be a soft tissue metastatic tumor. ${ }^{6}$

Histologic examination is paramount in confirming a definite diagnosis. Based on the histopathological finding, lesion was diagnosed as non-Hodgkin's lymphoma. Another diagnostic pitfall in connection with NHL as the possibility of its confusion with Hodgkin's lymphoma. The confusion arises mainly due to the presence of a typical multinucleated lymphocytic cells mimicking Reed-Sternberg like cells that characterize the Hodgkin's lymphoma. ${ }^{5}$ In such instance, the diagnosis is greatly facilitated by the accurate interpretation of immunohistochemical findings that differentiate nonHodgkin's lymphoma from Hodgkin lymphoma. The presence of positive staining for leukocyte common antigen CD-45, CD-20, BCL-20 and immunonegative for CD-30 and CD-3 distinguish the large B cell non-Hodgkin's lymphoma from Hodgkin's lymphoma. ${ }^{7}$ Correlation and interdependency of advance radiographic, histologic and immunohistochemical examination help in identify the disease in early stage, resulting in better prognosis.

\section{CONCLUSION}

Non-hodgkin lymphoma often shows an extranodal pattern, especially in the head and neck region. Different patient with primary extranodal location particularly in the oral cavity may be misdiagnosed. In addition to clinicoradiographic finding, additional examination used in this case is necessary for the early diagnosis and staging of cancer. So that more appropriate treatment can be instituted to the specific pathology.

\section{REFERENCES}

1. Kumar PP, Henchke UH, Kovi J. Malignant Lymphoma of the Floor of the Mouth. J National Med Assoc 1976 Jul;68(4): 300-302.

2. Agrawal MG, AgrawalSM, Kambalimath DH. Non-Hodgkin's lymphoma of maxilla: a rare entity. Natl J Maxillofac Surg 2011;2(2):210-213.

3. Jaffe ES, Harris, Stein NL, Isaccson PG. Classification of lymphoid neoplasm: the microscope as a tool for diseased discovery. Blood 2008 Dec 1;112(12):4384-4399.

4. Jayakrishnan R, Thomas G, Kumar A, Nair R. Non-Hodgkin's lymphoma of the hard palate. J Oral Maxillofac Pathol 2008 12(2):85-87.

5. Ramani P, Ahmed S, Janaki VR. Primary extranodal nonHodgkin's lymphoma of the oral cavity. Indian J Dermatol Venereol Leprol 2004;70(3):172-174.

6. Giri AK, Vishnu Vardhan GV, Bassi KK, Giri S. Non-Hodgkin's lymphoma of the infratemporal fossa: a case report. Indian J Cancer 2015;52(2):178-179. 
7. Higgins RA, Blankenship JE, Marsha C and Kinney. Application of immunohistochemistry in the diagnosis of Non-Hodgkin and Hodgkin lymphoma. Arch Pathol Lab Med 2008 Mar;132(3):441-461.

8. Shankland KR, Armitage JO, Hancock BW. Non-Hodgkin lymphoma. Lancet 2012 Sep 1;380(9844):848-857.
9. Isaacson $\mathrm{P}$, Wright DH. Malignant lymphoma of mucosaassociated lymphoid tissue - a distinctive type of B-cell lymphoma. Cancer 1983 Oct 15;52(8):1410-1416.

10. Cardona DM, Layne A, Lagoo AS. lymphomas of the gastrointestinal tract - pathophysiology, pathology, and differential diagnosis. Indian J pathol microbiol 2012;55(1):1-16. 